\title{
Method to Develop Functional Software for NPP APCS using Model-Oriented Approach in SimInTech
}

\author{
A.M. Shchekaturov， I.R. Kubenskiy， K.A. Timofeev， N.G. Chernetsov \\ Department of mathematical modeling \\ $3 \mathrm{~V}$ Services \\ Moscow, Russia \\ a.shchekaturov@3v-services.com
}

\begin{abstract}
The SimInTech environment developed in Russia enables using the end-to-end design of the algorithmic part of APCS for nuclear power plants (NPP), including the all-mode mathematical modeling of production process dynamics, debugging and optimizing control algorithms on an object model, generating functional software (FSW) as well as developing interfaces of the operator control panel. The article describes some of the main methods and approaches applied for the collective development of NPP APCS FSW. The implementation of the method during the development of APCS for Balokovo NPP-1 reactor compartment is presented as an example.
\end{abstract}

Keywords: model-oriented design, automated control system, algorithms, mathematical modeling, video frames

\section{Introduction}

A nuclear power plant, a ship with the NPP on board, and a submarine are all examples of the most complicated technical facilities produced by man. Designing a new NPP is always connected with a large scope of research and engineering activities, a group effort of specialist teams, the co-operation of several companies, and iterative calculations including those relating to optimization and search. In the process of NPP construction, external operating conditions should be considered along with the processes occurring inside the plant.

Automatic control systems (ACS) are an important NPP component that must comply with numerous, often contradictory, requirements. Control systems should feature a certain processing speed and a backup, ensure quality control and the required level of layered protection. Also, an option of manual control over production processes, an impactless mode-to-mode switch-over, the output of diagnostic information to the "upper" level, archiving signals with a specified stroke and others should be provided. It can be stated that all the specialists involved in the creation of APCS algorithms should know and understand thoroughly the physics of the phenomena, dynamics and technology of NPP processes.

As microprocessor technology develops, the rate of application of microprocessors in control circuits is increased; presently, we can discuss not only the control algorithms but also the functional software of ACS, which presents a non-trivial engineering problem. The complexity lies in the fact that not many process engineers can program, while programmers have a very little idea about technology. In general, process engineers articulate the ideas of algorithms in the language closest to them (including the functional block diagram language), whereas programmers interpret and implement these ideas in the way they have understood them, in a set of programs for specific equipment and the target operating system. At the same time, a large discrepancy may inadvertently appear between the software implementation conducted by the programmer and the initial ideas of the process engineer. It has to be eliminated at the stage of complex debugging and/or commissioning activities, which results in an increase in project costs and in a considerable loss of time.

In recent years, the model-oriented design (MOD) of control systems has been developed to a great degree (Voggenberger, 2005; Jakubowski, 2006; Dupleac, 2009) and has reached the level of a de facto standard to meet when generating FSW for ACS. Creating a mathematical model to describe the dynamics of a facility under design in a sufficient way forms the basis for the model-oriented approach. In the context of nuclear engineering, the MOD approach would be deemed optimal if it were not for technical complexities involved in its implementation, i.e. it is extremely difficult to create an all-mode model of NPP dynamics that would describe the dynamical characteristics of the facility in a satisfactory enough manner, it also represents single-purpose software that requires the application of design codes (thermo-hydraulic, neutronic, electrical, etc.). 


\section{The overall structure of the solution}

\section{SimlnTech allows to create software for upper and lower levels of I\&C system} SimlnTech provides remote debug, network data exchange, input of variables

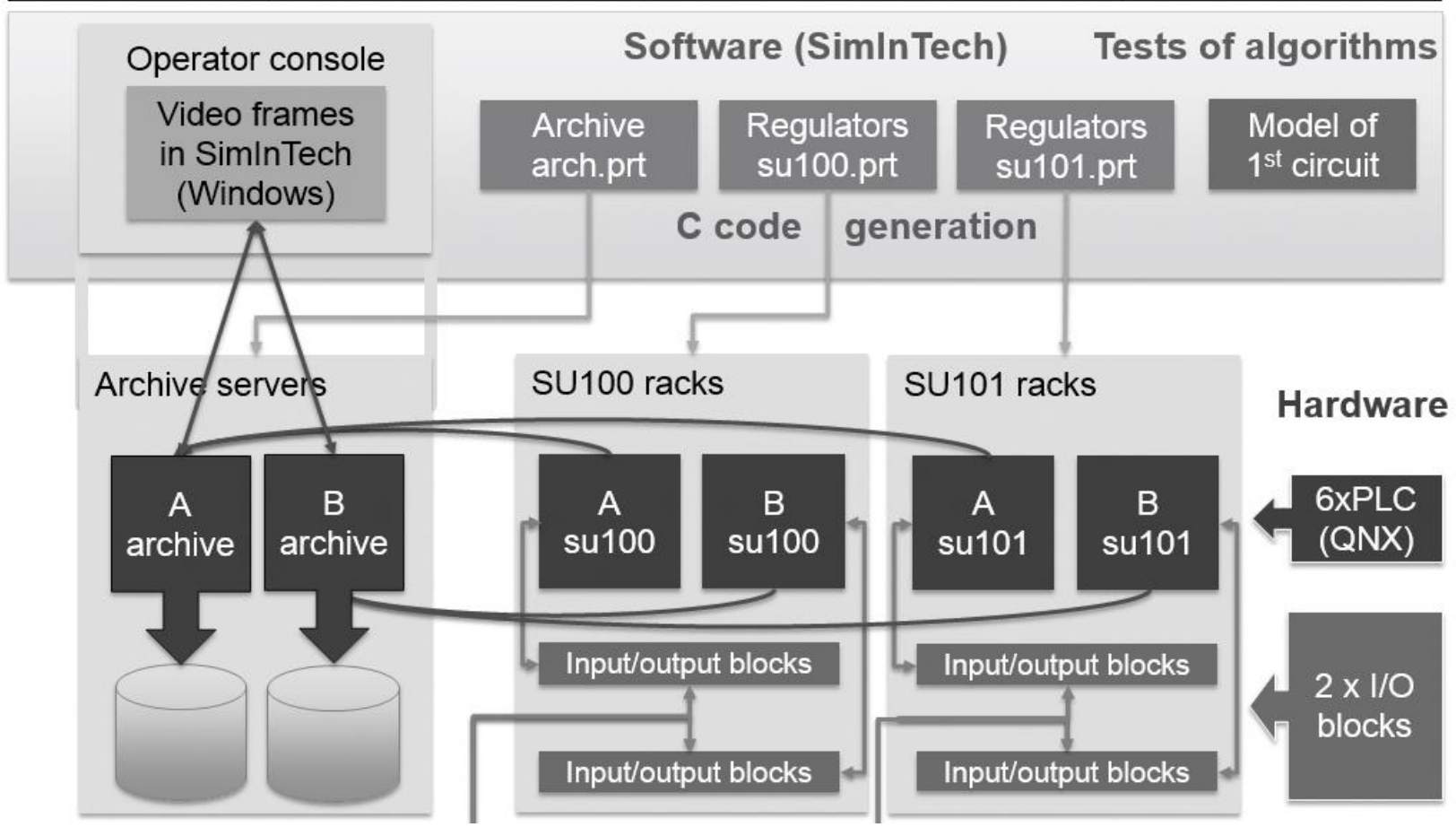

Figure 1. Hardware-Software Complex General View

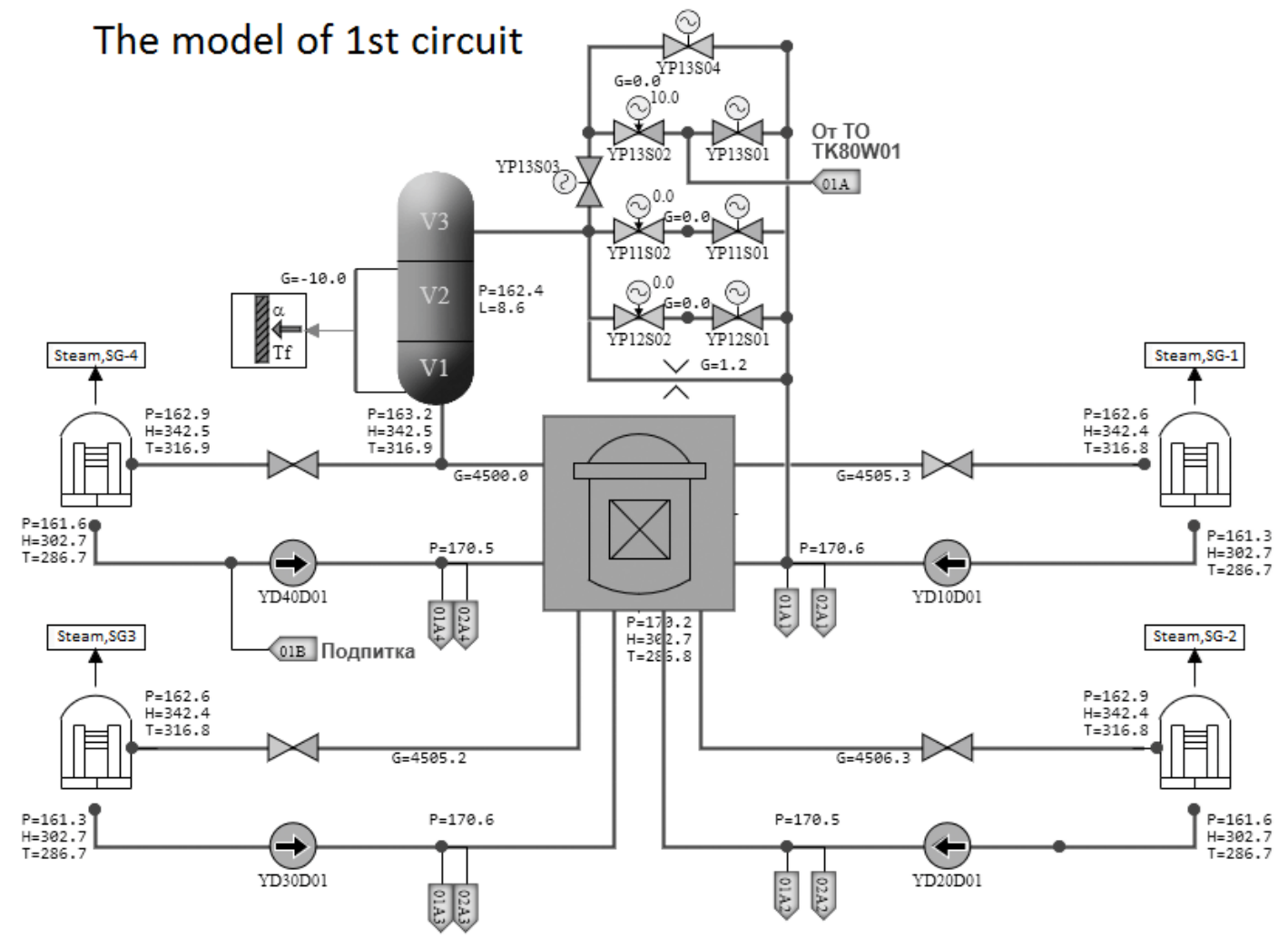

Figure 2. Design Diagram of the Primary Circuit Model 


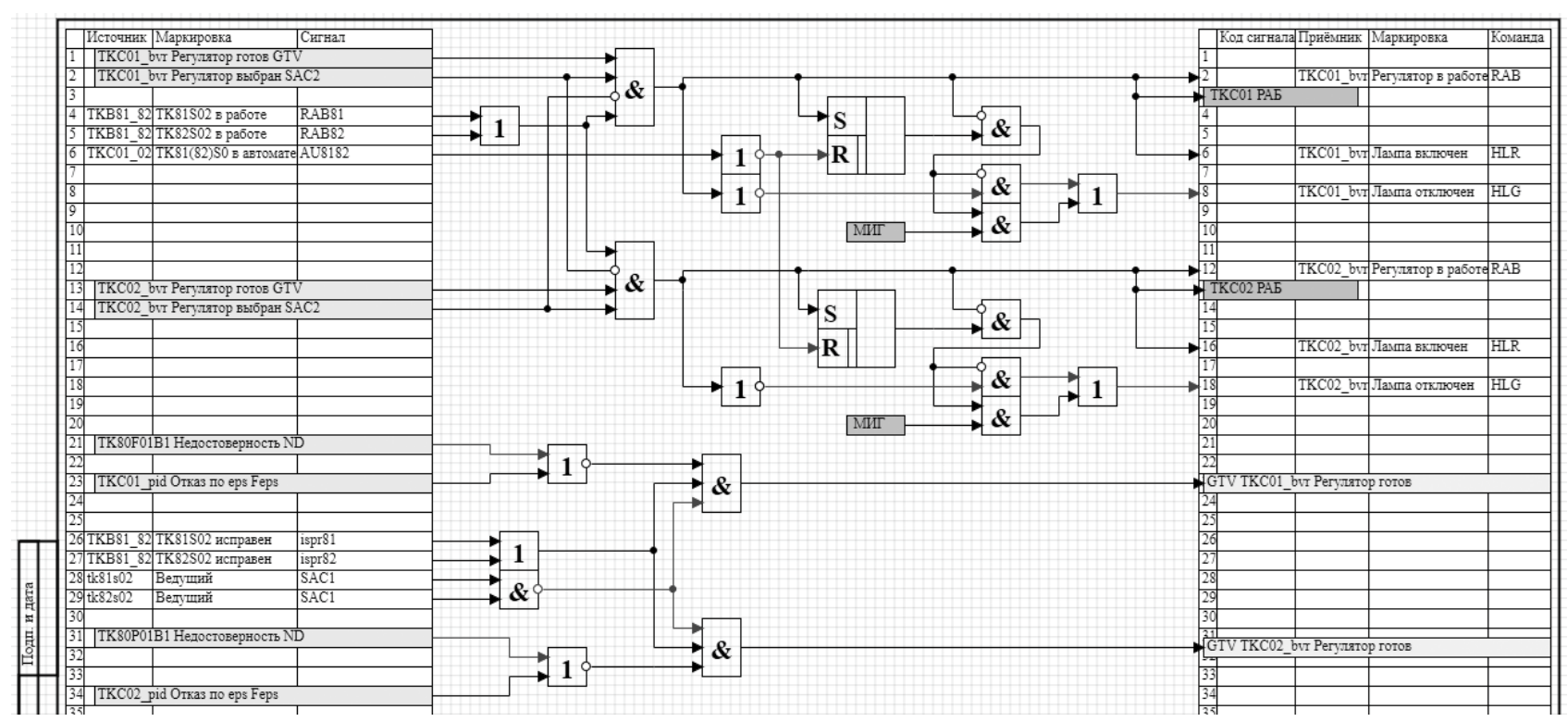

Figure 3. Example of a Functional Block-Diagram

Design codes are individual programs that have been developed for many years in design bureaus. Historically, they have not been developed for the purpose of integration and concurrent calculations with the use of any CAD of ACS algorithms. The SimInTech environment al-lows eliminating the problems relating to facility models and the integration of control algorithms thanks to its modular architecture, available interfaces to different design codes, and the relative simplicity of connection among new codes. The above fact has enabled the implementation of the "end-to-end" de-sign of the algorithmic part of APCS (Baum, 2012; Kozlov, 2005), i.e. control algorithms designed by technologists in the form of functional block-diagrams that are checked, debugged, and verified on an adequate model of facility dynamics are transmitted by the method of automatic generation of a code to the target sys-tem in the control equipment. As a result, prior to the stage of commissioning works, we obtain a product with verified FSW that is ready to be installed on the facility and requires virtually no adaptation or alteration.

\section{Methods to Develop APCS FSW in SimInTech}

The experience of applying the SimInTech environment to different pro-jects of mathematical modeling of NPP dynamics (Parshikov, 2013; Bezlepkin, 2013; Bolnov, 2014) and developing control algorithms evidentiates the main methods and techniques required for the development of ACS FSW. SimInTech authors believe that the sequence below can be of most use:

- $\quad$ stating the problem, identifying modeling and control boundaries, generating a list of equipment and signals, decomposing the facility into sub-systems, identifying boundary conditions; articulating agreement by the names of signals and variables;

- creating autonomous models of the physical processes occurring in the facility (models of processes of different physical nature, models of typical and unique pieces of equipment, models of actuators and devices, models of sensors, instruments);

- integrating the models into a complex model of dynamics of the facility;

- designing control algorithms for the facility in the form of functional block-diagrams, designing and modeling control panels and consoles;

- calculating concurrently and modeling facility dynamics and control system; testing and debugging the algorithms on the model, selecting and optimizing regulator coefficients;

- designing an analysis of the transient modes for normal operation and checking the operation of the automatic control algorithms;

- modeling design-basis and beyond-designbasis accidents as well as similar transient processes along with applying different equipment failures; adapting the algorithms;

- transmitting the control algorithms to the controllers, remotely debugging their implementation on the model;

- testing control equipment using testing devices which connect the mod-el as a simulator of the controlled facility by means of the inverse (digital-analog) transformation. 


\section{Applying SimInTech to Create Hardware-Software Complex of Automatic Regulation Facilities of Normal Operation Systems of Reactor Compartment}

The hardware-software complex of the automatic regulation facilities of the normal operation systems of the reactor compartment provides the automatic regulation of the main process parameters of the first circuit: the coolant pressure in the primary circuit above the reactor core, the water level in the pressure compensator, makeup pump flow rates and other parameters. HSC also generates signals for complex measurements of such process parameters as the maximum mean coolant temperature in the primary circuit circulation loops, a difference of the primary circuit saturation temperature and the maximum temperature in the hot threads of loops, the material balance of the coolant for flushing-makeup of the primary circuit and others. Some rather strict requirements were specified for the system in terms of processing speed (algorithm stroke and parameter archiving - no longer than $20 \mathrm{~ms}$ ), the number of registered and archived signals (over 15,000) and development periods (less than a year).

SimInTech has been used as the basis for developing a mathematical model of primary circuit dynamics (calculated by means of the TPP code), control and regulation algorithms, archiving server algorithms, a set of video frames and a system with analog input (see Figure 1). The code generator and executive environment included into SimInTech allowed the controllers to be programmed under the QNX real-time operating system, remote debugging of algorithm execution in the controllers to be carried out, and signals to be archived and displayed in video frames. The peculiarities of applying SimInTech to create HSC ARF RC are presented by implementing it in an integral design software environment for the upper and lower levels, including archiving system and system adjustment video frames:

- algorithm stroke - $10 \mathrm{~ms}$;

- registration of 7,500 signals in the archiving system;

- back-up of the controllers and input/output channels;

- regulator parameter input system.

The project dealt with the challenge of modernizing the available automatic regulation facilities of the reactor compartment along with replacing the APCS equipment implemented on a basically different hardware platform. Available control algorithms presented as a text and block-diagram images were used as reference data. The sequence for creating automatic regulation facilities included:
1. Developing a mathematical model of the reactor compartment in a scope sufficient for debugging the algorithms and the primary adjustment of the control regulators (Figure 2).

2. Developing design diagrams of the algorithms and regulators (see Figure 3).

3. Testing the algorithms on the mathematical model of NPP RC, calculating all the required modes of operation (reactor start and shutdown, primary circuit heating-up and cooling-down, reactor power increase and reduction, nominal mode of operation).

4. Developing the algorithms for input-output signals digital processing - checking the authenticity of a signal, diagnosing the transducer off-scale sweep or breakout, the overrun of the tolerable speed of a signal change, filtration, signal conversion to a physical value and the required units of measurement.

5. Binding process signals to input-output blocks, generating the initial code automatically, programming the controllers, testing and debugging the execution of the algorithms on the controllers.

6. Testing ARF devices on the facility model.

7. Programming archiving servers, developing the algorithms of control channel "equalization" (setting the slave channel in compliance with the status of the master control channel in case of a restart of the controller).

8. Developing a set of video frames including the illumination of the in-put of regulator parameters in the process of operating the controller (Figure 4).

This stage of commissioning works demonstrated an almost total absence of considerable errors- they were eliminated at the preliminary stages of developing and testing the algorithms.

\section{Conclusions}

Applying the SimInTech environment allowed the process engineers, programmers and test-operators of APCS to combine their efforts in an integral system, which led to a considerable (up to two-fold) reduction of the time required for developing a general software and hardware-software solution; it also minimized a number of errors in algorithm designing due to their elimination in the process of debugging on the facility model.

The application of this method in subsequent projects and in similar works by other teams has demonstrated the major practical advantage achieved due to the end-to-end design of control algorithms using the model-oriented approach. It is important to emphasize that the method developed on the basis of fully domestic tools provides as follows:

- exclusion of distortions in design solutions and their wrong interpretation by different groups of FSW designers (the minimization of the human factor); 


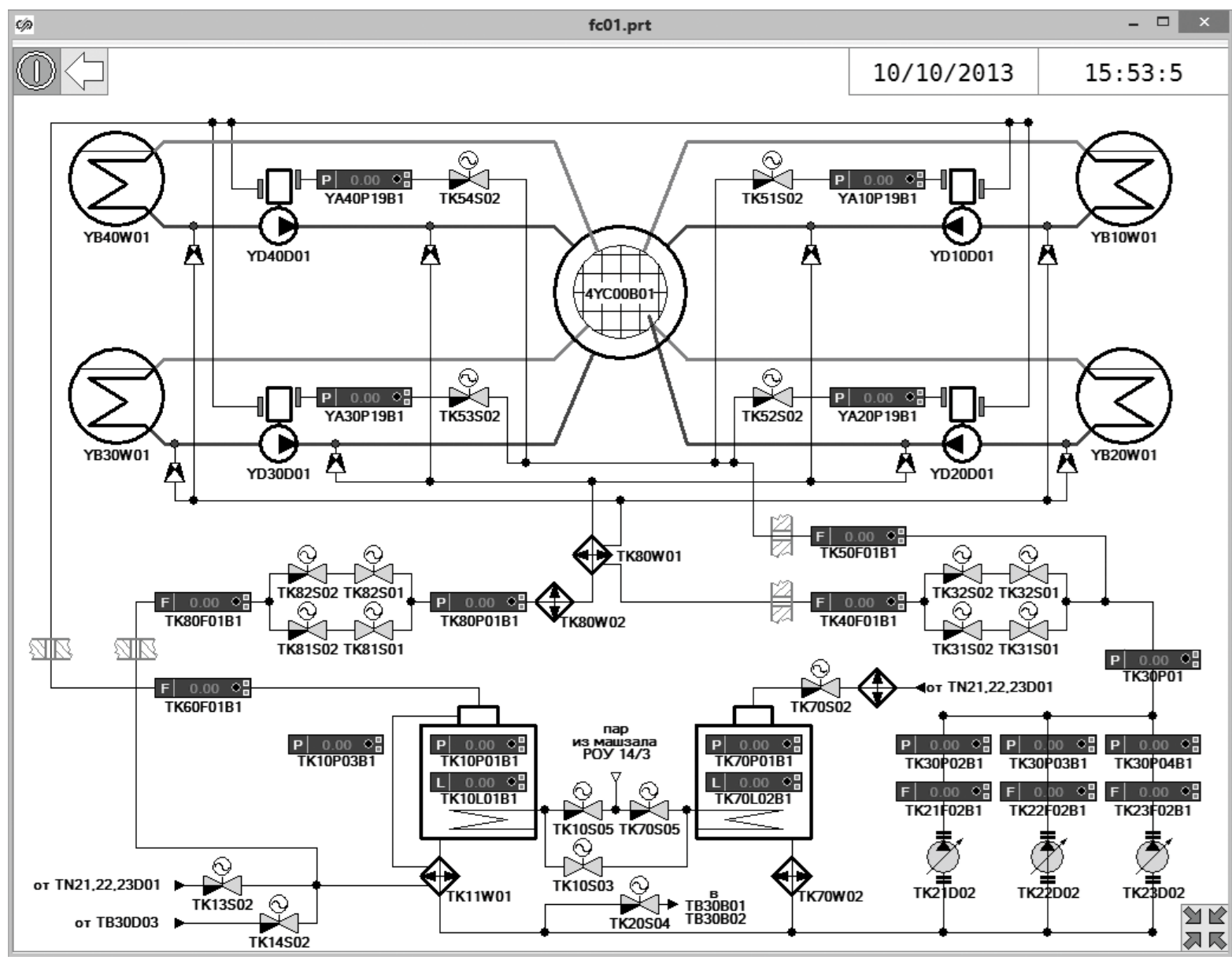

Figure 4. Example of Video Frame

- visualizing and debugging control system algorithms (reductions in the time for testing and debugging the instrument operation algorithm); a functional blockdiagram is both a design model and a component part of FSW documentation;

- automated design testing of control system algorithms using mathematical models of the controlled object (reductions in labor costs allocated to programmers and process engineers);

- data systematization and structuring as per control system algorithms during the whole life cycle of the facility: from the development of the terms of reference up to the operational period.

\section{References}

F. I. Baum, O. S. Kozlov, I. A. Parshikov, V. N., Petukhov, K. A. Timofeev, A. M. Shchekaturov, SimInTech Software for Programming Control System Instruments. Atomnaya Energiya (Atomic Energy) Magazine, 113, July-December, 2012.

V. V. Bezlepkin, V. O. Kukhtevich, E. P. Obraztsov, Yu. A. Migrov, Hardware-Software Complex "Virtual NPP unit with VVER" (HSC "VPU") for Testing Design Solutions NPP-2006. In the 8th International Scientific and Research
Conference "Provision of Safety for NPP with VVER", OKB "Gidropress", Podolsk, Russia, May 28-31, 2013.

V. A. Bolnov, I. S. Zotov, S. A. Malkin, A. S. Ushatikov, Experience of Creation of Modeling Complex for NPP with RU BN-1200. Atomny Proyekt (Atomic Project) Magazine, 19, October 2014

D. Dupleac, M. Mladin and I. Prisecaru, Generic CANDU 6 Plant Severe Accident Analysis Employing SCDAPSIM/RELAP5 Code, Nuclear Engineering and Design, 239: 2093-2103, 2009.

Z. Jakubowski, P. Dräger, W. Horche, W. Pointner, Development of Nuclear Plant Specific Analysis Simulators with ATLAS, In proceedings of the 6th Conference on Nuclear Option in Countries with Small and Medium Electricity Grids Dubrovnik, Croatia, 21-25 May 2006.

O. S. Kozlov, K. A. Timofeev, V. V. Khodakovsky. Software complex for research and development of dynamics and for designing of technical systems. Information technology, 9, 2005.

I. A. Parshikov, V. N., Petukhov, K. A. Timofeev, A. M. Shchekaturov, Modeling of Liquid-Metal Coolant Power Unit in SimInTech Software Complex. University Scientific Magazine, 5, 2013.

T. Voggenberger, D. Beraha and F.Cester, Nuclear Power Plant Simulation and Safety Analysis with ATLAS, The 16th IASTED International Conference on Modelling and Simulation MS 2005, May 2005, Cancun, Mexico 